\title{
Investigation of 3D particle flow in a flighted rotating drum
}

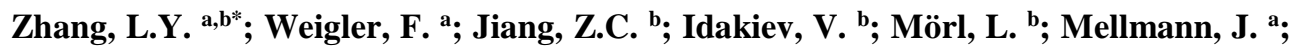 \\ Tsotsas, E. b;
}

a Department of Postharvest Technology. Leibniz Institute for Agricultural Engineering and Bioeconomy, Potsdam, Germany.

${ }^{\text {b }}$ Faculty of Process \& Systems Engineering. Otto-von-Guericke University Magdeburg, Magdeburg, Germany.

*E-mail of the corresponding author: lzhang@atb-potsdam.de

\begin{abstract}
To validate the particle motion in flighted rotating drum (FRD), a laboratory FRD was built and operated at 15\% filling degree and 10 rpm rotation speed using plastic balls as bed material. The particle tracking velocimetry (PTV) and magnetic particle tracking (MPT) techniques were applied to investigate the particle flow behavior. The $3 D$ particle flow was modeled by Discrete Element Method (DEM) with LIGGGHTS. The height of the barycenter for overall particles and particle instantaneous velocity were calculated from PTV and DEM data. The 3D time-averaged particle velocity distribution obtained from MPT experiment and DEM simulation was compared.
\end{abstract}

Keywords: flighted rotating drum; particle motion; particle tracking velocimetry; magnetic particle tracking; DEM simulation. 


\section{Introduction}

Flighted rotary drum (FRD) has been widely used in food and mineral industries for drying or cooling processes, as flights lift up particles and give them a higher chance to contact with gas. In last decades, much work has been carried out on FRD including particle mixing behavior, residence time distribution and discharge characteristics of flights [1]. However, few papers reported on particle movement, which is closely related to drying or cooling performance of drum. This is perhaps due to the limitation of experimental conditions and the complicated particle motion.

In order to investigate the characteristics of particle movement in FRD, particle tracking velocimetry (PTV) technique was utilized in current work to provide detail information of particle distribution and instantaneous velocities. Moreover, the magnetic tracking technique (MPT) was applied to evaluate particle velocity distribution in 3D. To simulate the particle motion, a 3D DEM model was developed, and the results obtained were compared with experimental data.

\section{Materials and Methods}

\subsection{Experimental setup}

The experimental setup consisted of a laboratory FRD, a high-speed camera system, and a magnetic tracking system as Fig. 1 schematically depicts.

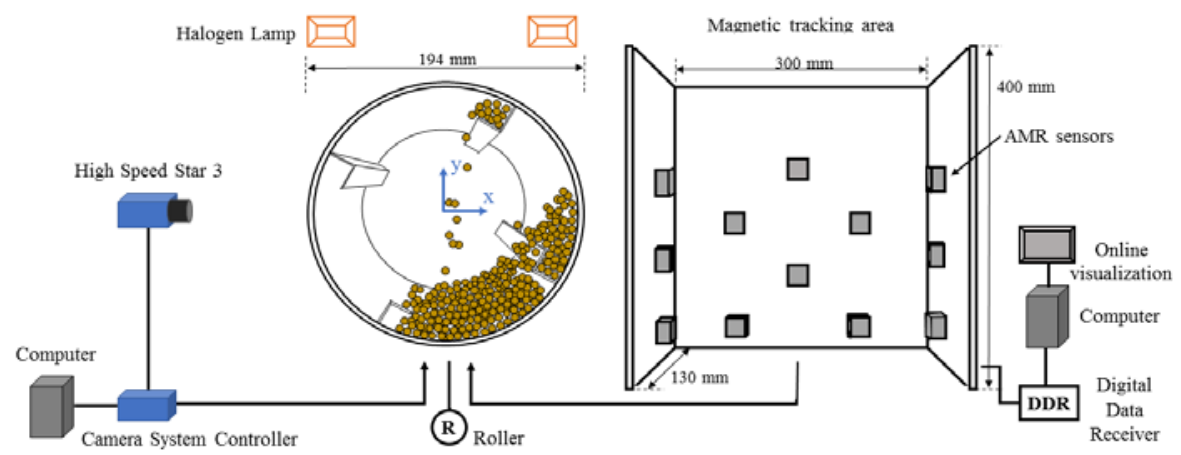

High speed camera system Flighted rotating drum

Magnetic particle tracking system

Fig. 1 Sketch of experimental setup, including a flighted rotating drum (middle), a high-speed camera system (left) and a magnetic particle tracking system (right).

\subsubsection{Flighted rotating drum (FRD)}

Figure 1 shows the horizontally placed drum with inner diameter of $194 \mathrm{~mm}$ and length of $150 \mathrm{~mm}$. Four rectangular flights were installed with a flight-length-ratio of 1.0. Fine sand (0.1 - $0.3 \mathrm{~mm}$ ) was glued on inner wall to prevent particle slippage; but end walls were kept 
smooth and transparent. The drum was made of acrylic glass, flights were made of aluminum. Spherical plastic balls were used as bed material with a diameter of $d_{p}=6 \mathrm{~mm}$ and a bulk density of $\rho_{b}=596 \mathrm{~kg} / \mathrm{m}^{3}$. The experiments were conducted at $15 \%$ filling degree (volume based) and $10 \mathrm{rpm}$ of drum rotating speed.

\subsubsection{High-speed camera system}

A high-speed camera system including a Photron high-speed camera (LaVision, CMOS chip) and a DaVis image acquisition software (LaVision) were used. Detailed parameters of the image system are listed in Table. 1 . Two continuous illuminating $400 \mathrm{~W}$ halogen lamps

Table 1. Parameters of the image system.

\begin{tabular}{lll}
\hline \multicolumn{1}{c}{ Parameters } & \multicolumn{1}{c}{ Value } & \multicolumn{1}{c}{ Unit } \\
\hline Display resolution & $1024 \times 1024$ & pixel \\
Frame rate & 500 & $\mathrm{fps}$ \\
Exposure time & $1 / 11,000$ & $\mathrm{~s}$ \\
Dynamic range & 10 & $\mathrm{bit}$ \\
Local length & 60 & $\mathrm{~mm}$ \\
$f$-number & 2.8 & - \\
\hline
\end{tabular}

were utilized to provide sufficient light, as shown in Fig. 1. The camera was positioned in front of the drum and adjusted to get a full view field of drum with $200 \times 200 \mathrm{~mm}^{2}$. With this setting, an approximate spatial resolution scale factor $S_{f}$ of $5.17 \mathrm{pixel} / \mathrm{mm}$ was obtained from the standard geometry calibration process in the DaVis software. The experiment lasted $19.2 \mathrm{~s}$ with a number of 9600 images, which was sufficient to represent the periodicity of particle flow in the FRD. The experiments were repeated three times.

\subsubsection{Magnetic tracking system}

In the magnetic tracking system, 12 Anisotropic Magneto Resistive (AMR) sensors were installed on three acrylic glass plates, which provided a closed tracking volume of $300 \mathrm{~mm}$ $\times 400 \mathrm{~mm} \times 130 \mathrm{~mm}$ as shown in Fig. 1 . The sensors were arranged and calibrated to provide an optimal measurement of the magnetic field strength. All sensors were connected to a data acquisition device, which recorded data and sent them to a lab computer via USB interface. Online visualization and data evaluation were achieved by associated software 'MagDat' and 'MagCal', respectively.

\subsection{Measurement techniques}

\subsubsection{Particle tracking velocimetry}

The particle tracking velocimetry (PTV) is a non-intrusive, image-based measurement technique to acquire particle velocities. By combining particle segmentation and tracking algorithms, a large number of particles could be tracked simultaneously. 
The particle-mask correlation method was applied to identify and determine the positions (coordinates $\mathrm{x}$ and $\mathrm{y})$ of individual particles [2,3]. A template particle matrix $(\mathrm{m} \times \mathrm{m}$ pixels), a key parameter in this process, was defined with the size of $d_{p} \bullet S_{f}$ pixels (rounding down to the nearest even number). The value of template matrix was averaged from 20 template particles that were selected manually. Then, a tracking algorithm named probability relaxation method was utilized to establish particle trajectories by tracking particle positions in consecutive image frames [3, 4]. During this process, three radiuses the maximum displacement radius, neighboring radius and the quasi-rigidity radius - were defined as $1.2 \cdot U_{\max } \cdot S_{f} \bullet \Delta t, 1.0 \cdot U_{\max } \bullet S_{f} \bullet \Delta t$ and $0.12 \cdot U_{\max } \bullet S_{f} \bullet \Delta t$ pixels, to search candidate particles and neighboring particles in the $1^{\text {st }}$ or $2^{\text {nd }}$ frame. The $U_{\max }$ was calculated based on the particle velocity during free fall in gas.

\subsubsection{Magnetic tracking technique}

The magnetic monitoring is based on continuous tracking of the magnetic marker. The evaluation of the measured data provides accurate information about the movement of tracer and, thus, enables investigating particle motion in a dense granular bed [5]. In this work, a $5 \mathrm{~mm}$ long cylindrical neodymium-iron boron with diameter of $3 \mathrm{~mm}$ was used as the magnet. By embedding it into a plastic ball, the tracer kept the same shape and size with other particles. With a measuring frequency of $250 \mathrm{~Hz}$, the MPT system could record the Cartesian coordinates $\mathrm{x}, \mathrm{y}, \mathrm{z}$ of tracer every 6 ms. One experiment lasted 6 minutes and was repeated three times.

\subsection{Discrete element method (DEM) simulation}

In the current work, the open source software LIGGGHTS was adopted for the DEM simulation. The motion of each particle in the Lagrangian framework was calculated by the Newton's second law with ignoring the particle-fluid interaction due to the big density difference between particle and gas phase. Based on a filling degree of $15 \%$ in the experiment, 3474 particles were inserted into the simulation chamber as shown in Fig. 2.

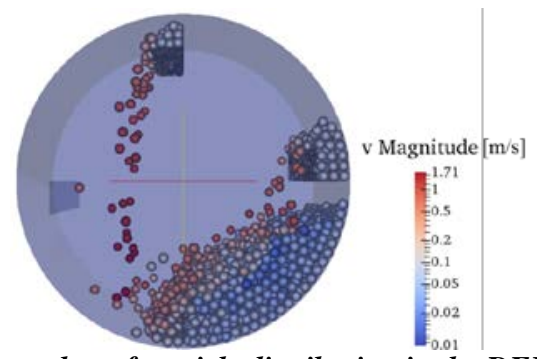

Fig. 2 Snapshot of particle distribution in the DEM simulation.

The particle interactions were calculated by the Hertzian contact model with tangential history tracking [6]. In this soft-sphere contact model, the effect of spring and dash-pot 
appear through stiffness $\kappa$ and damping coefficient $\eta$, which were determined by the physical material properties of Poisson ratio $\sigma$, Young's modulus $E$ and the coefficient of restitution $e$. The detailed material properties of particles are listed in Table. 2. DEM simulation was run for $120 \mathrm{~s}$ in total, but only from the last $30 \mathrm{~s}$ data was extracted at $0.002 \mathrm{~s}$ intervals for further analysis.

Table 2. Material properties and model parameters for DEM simulation.

\begin{tabular}{lll}
\hline \multicolumn{1}{c}{ Parameters } & \multicolumn{1}{c}{ Value } & \multicolumn{1}{c}{ Unit } \\
\hline Young's modulus $E^{[7]}$ & $10^{7}$ & $\mathrm{~Pa}$ \\
Poisson ratio $\sigma$ & 0.3 & - \\
Rolling coefficient $\mathrm{\kappa}_{\mathrm{r}}{ }^{[8]}$ & 0.01 & - \\
Coefficient of restitution $e^{[9]}$ & 0.68 & - \\
Friction coefficient $\mu_{\mathrm{f}}{ }^{[10]}$ & 0.2 & - \\
Time step $\Delta \mathrm{t}_{\mathrm{DEM}}$ & $5 \times 10^{-6}$ & $\mathrm{~s}$ \\
Simulation time $\mathrm{t}_{\mathrm{sim}}$ & 120 & $\mathrm{~s}$ \\
\hline
\end{tabular}

\subsection{Post-processing of experimental and simulation data}

\subsubsection{Barycenter calculation}

To describe the location of the overall particles in the FRD, the barycenter $r_{c}$ was used as calculated from Equation (1a). The r represents the particle coordinates value $\mathrm{x}, \mathrm{y}$ or $\mathrm{z}$. The $\mathrm{m}_{\mathrm{i}}$ denotes the mass of particle $\mathrm{i}$ and $\mathrm{n}$ the particle number. Considering that the plastic balls are regular spheres with uniform density, the equation is simplified as Equation (1b).

$$
r_{c}=\frac{\sum_{i=1}^{n} m_{i} \cdot r_{i}}{\sum_{i=1}^{n} m_{i}} \quad \text { (a); } \quad r_{c}=\frac{\sum_{i=1}^{n} r_{i}}{n} \quad \text { (b); }
$$

\subsubsection{Particles velocity calculation}

The particle instantaneous velocity was calculated from the particle coordinates $r\left(t_{2}\right)$ and $r\left(t_{1}\right)$ for the case $t_{2} \rightarrow t_{1}$ by Equation (2). With the spatial velocity components $v_{\mathrm{x}}, \mathrm{v}_{\mathrm{y}}$ and $\mathrm{v}_{\mathrm{z}}$, the total particle velocity can be determined by Equation (3) in 3D.

$$
\begin{aligned}
& v\left(t_{1}\right)=\lim _{t_{x} \rightarrow t_{1}} \frac{r\left(t_{2}\right)-r\left(t_{1}\right)}{t_{2}-t_{1}} \\
& v_{3 D}(t)=\sqrt{v_{x}(t)^{2}+v_{y}(t)^{2}+v_{z}(t)^{2}}
\end{aligned}
$$

\section{Results and Discussion}

\subsection{Barycenter migration}

Due to the pushing force of the flights, the position of the overall particles was raised increasing the particle-gas contact and, thus, heat transfer. Figure 3 represents the 
normalized Y coordinate of the barycenter for overall particles over time for two drum rotations (12 s duration) as obtained from the PTV measurement and DEM simulation. As the figure illustrates, the barycenter rotated every $1.5 \mathrm{~s}$ corresponding to a quarter of one rotation of the flights. Furthermore, the DEM data agreed well with the PTV measurement.

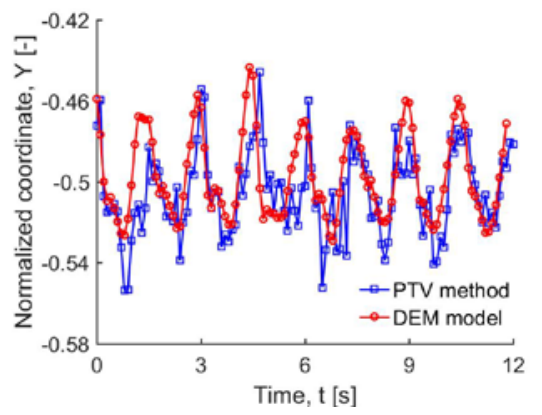

Fig. 3 Normalized $Y$ coordinate value of particles barycenter in $12 s$ duration time (two rotating circles) by PTV method and DEM model.

\subsection{Particle instantaneous velocity}

Figure 4 shows the particle instantaneous velocity fields from PTV measurement and DEM simulation for the flight positions of $0^{\circ}, 30^{\circ}, 60^{\circ}$ to the horizontal, respectively.
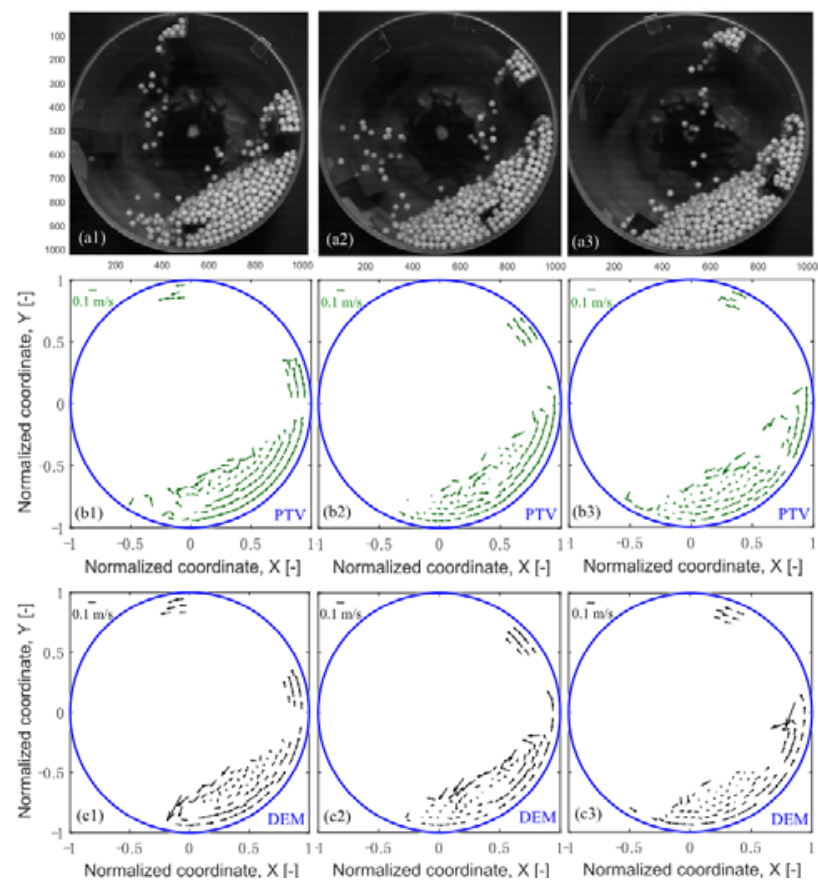

Fig. 4 Instantaneous particle distribution (a1-a3) and velocity vectors from PTV measurement (b1b3) and DEM model (c1-c3) at the time of 0 s (a1, b1, c1), $0.5 s(a 2, b 2, c 2)$ and $1.0 s(a 3, b 3, c 3)$. 
Since the PTV is limited to 2D studies, the particles located in the fist layer behind the front plate were chosen in DEM simulation to generate the particle velocity fields. The figures a1-a3 show the images of instantaneous particle distributions. The vortex in the dense phase at the bottom is clearly visible in Fig. 4 b1-b3 and c1-c3. The passive phase on flights presented a relatively homogeneous velocity distribution as they moved synchronous with the rotating wall. It is important to notice that, with different location of the flights, not only the position of vortex center dynamically changes but also the thicknesses of active and passive layers. This effect greatly accelerates the mixing of dense phase and, thereby, the heat transfer. The DEM data are in good agreement with PTV data.

\subsection{Particle velocity distribution}

Considering that PTV is limited to 2D, the 3D time-averaged and normalised velocity distributions were compared between MPT and DEM data in Fig. 5 to further validate particle motion in the FRD.
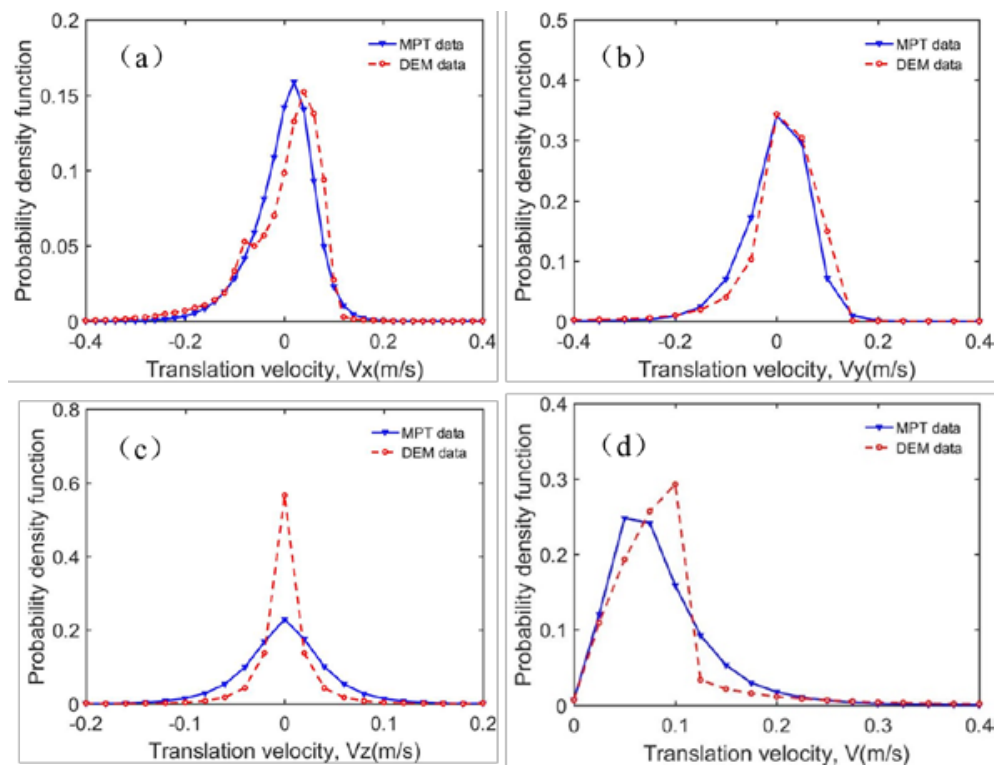

Fig. 5 Probability density distribution of particle translational velocity components by MPT measurement and DEM simulation: (a) Vx; (b) Vy; (c)Vz; (d) total velocity $V$.

It should be noted that the MPT data was obtained from 18 mins (three repeated experiments) to ensure a high accuracy of data distribution. While, due to computational limitations, the data from all particles (3,474 in total) were chosen from the last $30 \mathrm{~s}$ (5 rotating circles) of the DEM simulation for comparison. For radial velocity $\mathrm{Vx}$ and $\mathrm{Vy}$, very similar distributions were obtained from MPT and DEM data, see Fig. 5a, b. The curves are asymmetric in shape which may be due to the influence of flights. Whereas, the axial velocity distribution Vz shows symmetry (Fig. 5c). Furthermore, the DEM data display a narrower distribution and a sharper peak. Potential reason for this difference could be attributed to the collision model selected in DEM. The total velocity distribution of the 
MPT and DEM data show a comparable trend in lower velocity region, but apparent differences in higher velocity region, as shown in Fig. 5d. In general, there is reasonable agreement in the velocity distribution between MPT experiment and DEM model.

\section{Conclusion}

To investigate the particle motion in the FRD, two experiment techniques, PTV and MPT, were applied. The particle motion was modeled by using the DEM. A periodically upward movement of particles was confirmed. Moreover, the position of the vortex generated in dense phase varied over time, and the particle transient velocity distributions displayed asymmetric profiles. All these effects are caused by the flights. In addition, the DEM results agreed well with the PTV and MPT measurements.

\section{References}

[1] Delele, MA; Weigler, F.; Mellmann, J. Advances in the application of a rotary dryer for agricultural products: A review. Drying Technology 2015, 33 (5), 541-558.

[2] Takehara, K.; Etoh, T. A study on particle identification in PTV particle mask correlation method. Journal of Visualization 1998, 1 (3), 313-323.

[3] Jiang, Z.; Hagemeier, T.; Bueck, A.; Tsotsas, E. Experimental measurements of particle collision dynamics in a pseudo-2D gas-solid fluidized bed. Chemical Engineering Science 2017, 167, 297-316.

[4] Brevis, W.; Niño, Y.; Jirka, G. H. Integrating cross-correlation and relaxation algorithms for particle tracking velocimetry. Experiments in Fluids 2011, 50(1), 135147.

[5] Idakiev, V.; Mörl, L. How to measure the particle translation and rotation in a spouted and fluidized bed. Journal of Chemical Technology and Metallurgy 2013, 48(5), 445450.

[6] Tsuji, Y.; Tanaka; T., Ishida, T. Lagrangian numerical simulation of plug flow of cohesionless particles in a horizontal pipe. Powder Technology 1992, 71, 239-250.

[7] Chen, H.; Xiao, YG.; Liu, YL.; Shi, YS. Effect of Young's modulus on DEM results regarding transverse mixing of particles in a rotating drum. Powder Technology 2017, 318, 507-517.

[8] Goniva, C. C.; Kloss, N. G.; Deen, J. A.; Kuipers, M.; S. Pirker, Influence of rolling friction on single spout fluidized bed simulation, Particuology 2012, 10(5), 582-591.

[9] Jiang, Z.; Rieck, C.; Bueck, A.; Tsotsas, E. Estimation of coefficient of restitution of irregularly shaped particles on horizontal substrates, 8th International Granulation Workshop, Sheffield, UK, June 28-30, 2017.

[10] Santos, D. A.; Barrozo, M. A.; Duarte, C. R.; Weigler, F.; Mellmann, J. Investigation of particle dynamics in a rotary drum by means of experiments and numerical simulations using DEM. Advanced Powder Technology 2016, 27(2), 692-703. 